\title{
Likelihood Partitioned Bayesian Filtering for Lithium Sulfur Battery State Estimation
}

\author{
Srinivasan Munisamy, Senior Member, IEEE, Daniel J. Auger, Senior Member, IEEE, Abbas \\ Fotouhi, Member, IEEE, Bob Hawkes and Euthymios Kappos
}

\section{Post Conference Paper}

\begin{abstract}
This paper introduces a novel Bayesian filtering design framework for estimating the state-of-charge (SoC) and state-of-energy (SoE) of Lithium-sulfur (Li-S) batteries suitable for direct deployment in real-time battery management systems (BMSs). In Li-S batteries, the opencircuit voltage has two regions: a steeply-descending 'highplateau' and a flat 'low-plateau'. There is a sharp transition between the two. Because of this, battery parameter are segmented into two regions; high- and low-plateau parameters. In contrast with traditionally Bayesian approaches, each partitioned parameter set is given its own probability density functions (PDFs) for a priori state and likelihood and propagates any one of them at a time. The proposed strategy has been implemented with a simple nonlinear Bayesian filter, namely the extended Kalman filter (EKF). The performance of both SoC and SoE estimators is demonstrated with experimental test data for a Li-S battery cell designed for aerospace application with a pulse discharge duty cycle. The cell's experiment was considered under two different temperature conditions. The proposed likelihood partitioned EKF was validated under two different initial estimated state conditions. The proposed estimators gave good accuracy, achieving 97 percent.
\end{abstract}

Index Terms-battery management systems, Lithium sulfur battery, extended Kalman filter, nonlinear equations, nonlinear filters, parameterization, state estimation, state-ofcharge, state-of-energy.

\section{INTRODUCTION}

$\mathrm{N}$ recent years, research in the area of lithium-sulfur (Li-S) battery technology has accelerated the advances in chemistry [1-3], modeling [4] and battery management system [5-9]. The studies on the chemistry behaviors show that Li-S batteries are environmentally friendly, safe, and low cost as they use toxin-free

Manuscript received Month xx, 2xxx; revised Month xx, xxxx; accepted Month $x, x x x x$. This work was supported in part by the Aerospace Technology Institute (ATI) and Innovate UK who funded this work under grants TS/P003818/1 to ZIP and TS/R013780/1 to LiSFAB, respectively and European commission grant 814471.

S Munisamy, D J. Auger, A. Fotouhi and Neda Shateri are with the Advanced Vehicle Engineering Centre (AVEC), SATM, Cranfield University, Cranfield, MK43 OAL,UK (e-mail: and abundant sulfur cathode. In addition to these, the high energy density and cycle life of Li-S batteries are evidence that the Li-S batteries may be an alternative choice to lithium-ion (Li-ion) batteries for aerospace and automotive applications. Battery management systems (BMS) is an essential component of the Li$\mathrm{S}$ battery powered propulsion system.

In BMS of Li-S batteries, the estimation of Li-S battery's performance metrics accurately is a challenging and complex problem as the electrical characteristics of Li-S batteries are highly nonlinear and dynamic. Particularly, the discharge opencircuit voltage of Li-S is a highly nonlinear function, which has a deep-dive high-plateau, range from 2.6 to $2.1 \mathrm{~V}$ and in flat lowplateau which has poor observability [7], range from is 2.0 to 1.4 $\mathrm{V}$. In addition to the slope of high plateau of voltage changes, the joining point and equivalent circuit network (ECN) model parameters are sensitive to the temperature $[8,14]$. Propp et.al [6] used Bayesian filtering techniques, including extended Kalman filter (EKF), unscented Kalman filter (UKF) and particle filter (PF) for state-of-charge (SoC) estimation. In [9] the dual EKF proposed for based SoC, the state of available Power (SoAP) and state of health $(\mathrm{SoH})$. On other hand, Abbas et.al [7] presented Li$\mathrm{S}$ battery SoC estimation using adaptive neuro-fuzzy inference system (ANFIS) with PEM based ECN parameterization, and [4] presented support vector machine (SVM) based SoC estimation of Li-S batteries with weighted least-square ECN parameterization.

Results of the above literature were promising However, further research on Li-S battery management (BMS) needs to overcome the limitations. For example, training and mapping with the Coulomb counting approach in ANFIS and SVM, lead to a significant computational issue. The accuracy of Li-S battery's SoC estimation by both ANFIS [7] and SVM [4] improved when their response was merged to the Coulomb counting SoC, which is impractical to evaluate accurately when the initial $\mathrm{SoC}$ is unknown. Compared to machine learning algorithms, the

srinivasan.munisamy@cranfield.ac.uk;d.j.auger@cranfield.ac.uk;a.fotouhi@cran field.ac.uk).

Bob Hawkes, and E. Kappos are with Oxis Energy, Abington, Oxfordshire, OX14 1RL, UK (email: Bob.Hawkes@oxisenergy.com; Euthymios.Kappos@oxisenergy.com )

Corresponding author's phone: xxx-xxx-xxxx; fax: xxx-xxx-xxxx; email: srinivasan.munisamy@cranfield.ac.uk). 
nonlinear filters are very simple two-step recursive processes, estimating the state based on the measurement and easy to implement for real-world applications. Nevertheless, the conventional Bayesian approaches [13] used a partial sinusoidal function (PSF) for smooth transition of high to low-plateau of Li$\mathrm{S}$ battery parameters. To eliminate PSF, in conference paper [11] a modified filtering strategy, with likelihood-partition, was proposed for SoC and SoE estimation of Li-S battery. Hereafter the likelihood-partitioned filtering approach is referred as a likelihood-partitioned Bayesian filter (LPBF).

This paper is an extended version of [11]. In [11], the results of proposed approach for SoC and SoE estimation of Li-S cell at $20^{\circ} \mathrm{C}$ temperature under a single initial estimated state condition. Contrast to conference, this article briefs the concept and theory of the LPBF for SoC and SoE estimation of Li-S battery cell. This paper also presents improved estimation results of proposed approach after recalibrated it. Furthermore, this work addresses the battery surface temperature issue by studying performance at two different temperature, for instance, $20^{\circ} \mathrm{C}$ and $30^{\circ} \mathrm{C}$. In addition, this version investigates reliability of LPBF by assuming two different initial estimated state. Having experimental pulse drive cycle test data set, the SoC and SoE estimation of Li-S by proposed LPBF is validated. Results show that the proposed method outperforms and accurately estimates SoC and SoE of Li-S battery cell, without large training and additional computation burden.

\section{BATTERY EXPERIMENT AND MODELLING}

This section presents experimental test data (a link for data access is give in the acknowledgement) and explains how such data can be modelled and used to estimate the SoC and SoE of $\mathrm{Li}-\mathrm{S}$ batteries. As test data is the required information to the filterbased state estimation, the section starts with sensor data and then briefed the battery's ECN model in terms of SoC and SoE.

\section{A. Experimental Battery Sensor Data}

The battery experiment test rig-measurement system provides the current, voltage and surface temperature. Fig 1 shows a highlevel black diagram for test rig, sensor data flow and their integration to the filter based $\mathrm{SoC}$ and SoE estimators.

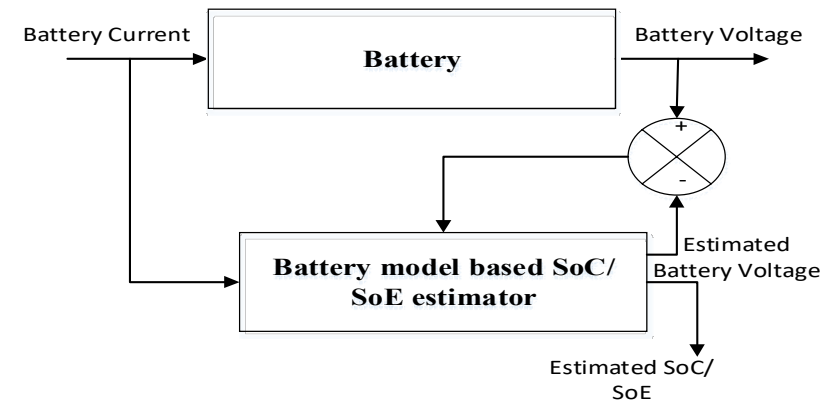

Fig. 1. Block diagram of experimental battery rig with input current and output voltage that used by proposed Li-S battery state estimation.

This test rig integrates source and load, where computer control system controls and emulates the real-world operating conditions of battery cell. A small unmanned air vehicles (UAV) application designated type of Li-S battery cell is used for experiment. Table
1 shows the important specifications the cell. As shown in the first column of Table 1, the capacity of Type A cell is 1.9Ah. From the total energy and mass density given in the table I, it is an evident that the energy density is about $120.25 \mathrm{Wh} / \mathrm{Kg}$.

TABLE I

\begin{tabular}{lll}
\multicolumn{2}{c}{ SPECIFICATION OF LI-S BATTERY CELLS USED FOR EXPERIMENT } \\
\hline \hline \multicolumn{1}{c}{ Specification with unit } & \multicolumn{1}{c}{ specifications } \\
\hline Capacity (Ah) & 1.9 \\
Total energy (Wh) & 5 \\
Maximum voltage (V) & 2.6 \\
Minimum voltage (V) & 1.4 \\
Nominal voltage (V) & 2.1 \\
Maximum discharge current (A) & 6 \\
Maximum charge current (A) & 1 \\
Cell mass (kg) & 0.0158 \\
\hline \hline
\end{tabular}
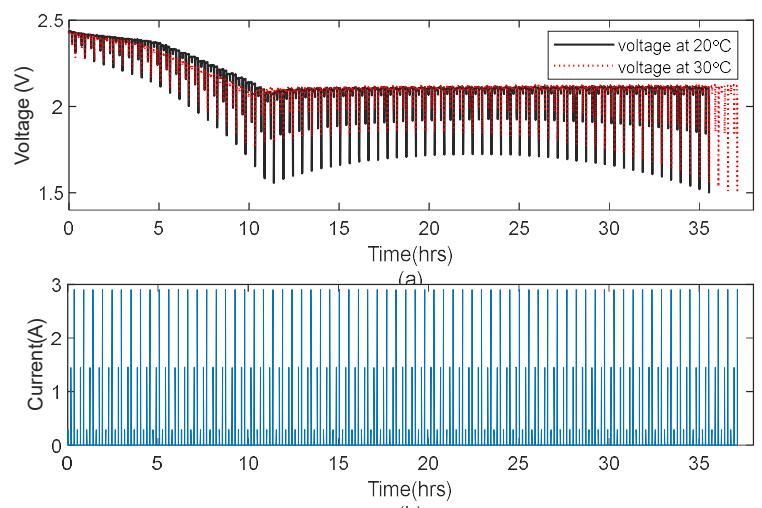

Fig. 2. Test data of type A cell; (a) Battery terminal voltage, (b) load current (negative magnitude indicates discharge current).

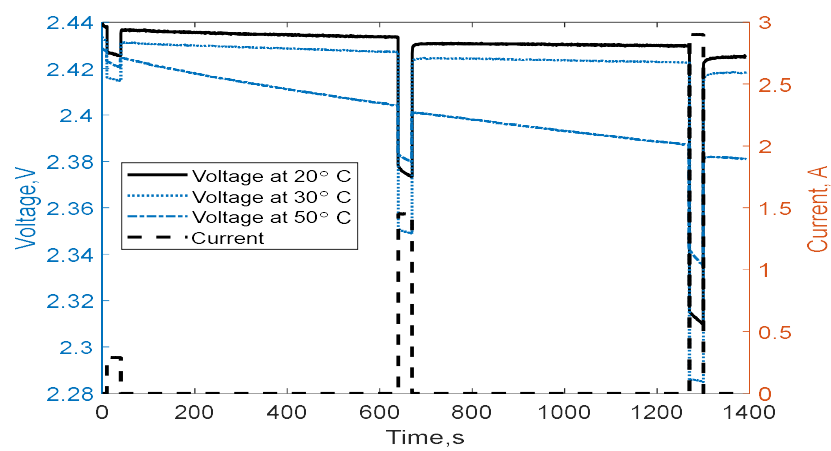

Fig. 3. Change in response of Li-S battery cell to short-period current pulses as temperature changes

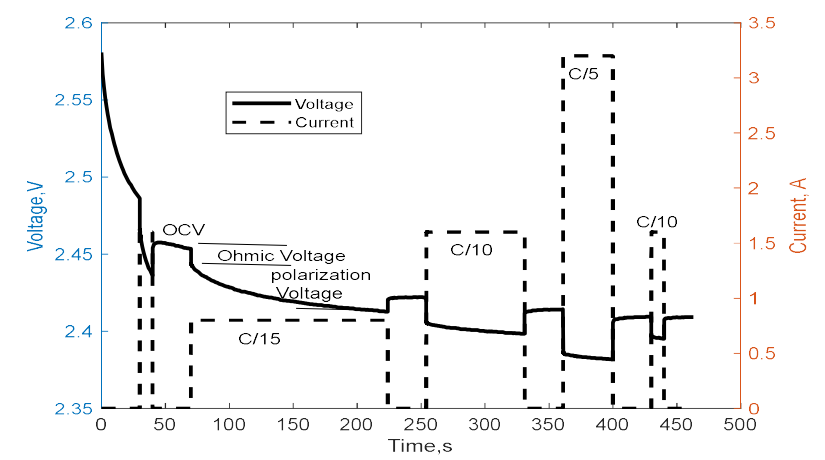

Fig. 4. Voltage response of Li-S battery cell to different C-rate

Fig 2 shows test data of Li-S cell discharge cycles test at two different temperature. For instant, Fig 2a shows the measured 
battery voltage at temperature $20^{\circ} \mathrm{C}$, and $30^{\circ} \mathrm{C}$ for current pulses of mixed C-rate. In Fig $2 \mathrm{a}$, the black solid line denotes the voltage response at $20^{\circ} \mathrm{C}$ whereas the red-dotted line shows the response at $30^{\circ} \mathrm{C}$. We have conducted experiment for $50^{\circ} \mathrm{C}$, voltage response of Li-S cell shown by blue-dotted line of fig 3., but it is not discussed and used in this paper for the sake of simplicity. Fig $2 \mathrm{~b}$ shows the mixed C-rate current pulses that applied to the $\mathrm{Li}-\mathrm{S}$ cell. It is noted that duration of pulses is fixed and short. This may be best fit to drive cycle of UAV application [10]. Test results show that the voltage of Li-S battery is nonlinear in nature, that is, deep-dive high-plateau and flat low-plateau. Furthermore, the voltage characteristic of $\mathrm{Li}-\mathrm{S}$ cell varies as the temperature varies, for instance, the voltage at $30^{\circ} \mathrm{C}$ is slightly lower than the voltage at $20^{\circ} \mathrm{C}$ as shown in Fig 3. For study the dynamic characteristic such polarization voltage, we have conducted experiment with $\mathrm{C} / 15$ rate current pulses $200 \mathrm{~s}$ length and observed the voltage response, which is shown as Fig 4. Fig 4 shows that Li-S cells are highly dynamic [8] to C-rate of current level. These are challenging to model and estimate battery state. The battery state and dynamic model of Li-S cells are briefed in the next section.

\section{B. Battery Modeling}

The battery output voltage is a function of the OCV, the voltage across the internal resistance and dynamic polarization voltage. Since the measured voltage is not only function of current and temperature, it is also a function of state of battery. therefore, the ECN model for SoC estimation problem is a function of SoC as shown Fig 5. Similarly, the ECN model of Li-S cell for SoE problem is expressed as function of SoE as shown in Fig 6.

\section{B. Battery states}

This subsection describes the SoC and SoE of battery, and how each parameter of 1RC ECN models is expressed as a function of each such state. Because [7] defined the integration of current is SoC whereas [15] defined the integration of power as SoC, this study explores the significant difference, if any, between $\mathrm{SoC}$ and SoE, their challenges and advantages in Li-S BMS by analysing and estimating SoC in Ampere-hour (Ah) and SoE in Watt-hour (Wh) and their normalization. The estimation of SoE in Wh can be directly used for optimize the energy and predict the remaining time of the battery use [12]. It is assumed that both state and parameters have no parameter uncertainty caused by temperature change.

The SoC can be evaluated by integrating the current. After converting the integration into summation, the SoC in Amperehour (Ah), is expressed as

$x_{s o c}(k)=x_{\text {soc }}(k-1)-I_{L}(k) \Delta t+\Delta x_{\text {soc }}(k)$

or the $\mathrm{SoC}$ in normalization is expressed as

$x_{\text {soc }}(k)=x_{\text {soc }}(k-1)-\frac{I_{L}(k) \Delta t}{C_{B}}+\Delta x_{s o c}(k)$

Where

$x_{\text {soc }}(k)=f_{c, 1}\left(x_{c}(k-1), I_{L}(k)\right)+\Delta x_{s o c}(k)$

is the $\mathrm{SoC}$ at instant $k, C_{B}$ is the total capacity, unit $\mathrm{Ah}$, of the battery, $\Delta x_{\text {soc }}(k) \sim N\left(0, Q_{x}\right)$ is a Gaussian noise, which is an error due unknown modelling or due integral approximation, $\Delta t=T_{S}$ denotes the sampling time in seconds.

Like SoC, the SoE can be evaluated by integrating power as $[11,12]$. Therefore, the SoE in Wh with unknown modelling error can be expressed as $x_{\text {soe }}(k)=x_{\text {soe }}(k-1)+V_{\text {ocv,soe }}(k) I_{L}(k) \Delta t+\Delta x_{\text {soe }}(k) \quad$ (3a) where $V_{o c v, s o e}(k)=V_{o c v}\left(x_{s o e}\right)$ is the open-circuit voltage, which is a function of SoE.

The SoE in normalization with unknown modeling error can be written as

$x_{\text {soe }}(k)=x_{\text {soe }}(k-1)+\frac{V_{o c v, s o e}(k) I_{L}(k) \Delta t}{E_{B}}+\Delta x_{\text {soe }}(k)$

This can be expressed as

$x_{\text {soe }}(k)=f_{e, 1}\left(x_{e}(k-1), V_{o c v, \text { soe }}(k), I_{L}(k)\right)+\Delta x_{\text {soe }}(k)$

where $\Delta x_{\text {soe }}(k) \sim N\left(0, Q_{x}\right)$ is a Gaussian noise with mean zero and variance $Q_{x}$. where $E_{B}$ is the energy capacity of the battery cell, this is not same as total capacity of battery cell $C_{B}$.

\section{Polarization Voltage}

The polarization voltage across $\mathrm{RC}$ network is given as [1-3] $\frac{d V_{P}(t)}{d t}=-\Omega(.) V_{P}(t)+\rho(.) R_{\text {int }}(.) \Omega(.) I_{L}$

where

$\Omega()=.\frac{1}{R_{P}(.) C_{P}(.)}$

$R_{\text {int }}()=.R_{i}()+.R_{P}($.

$\rho()=.\frac{R_{P}(.)}{R_{\text {int }}(.)}$

The parameters form vector $\theta()=.\left[\mathrm{V}_{\text {oc }}(.), \mathrm{R}_{\text {int }}(.), \Omega(.), \rho(.)\right]^{\prime}$ These are related the actual ECN parameters that given in fig 5 of SoC and Fig 6 of SoE characterization. The dynamics characteristics of Li-S battery cell are studied by polarization voltage.

The dynamic of polarisation voltage $V_{P}(t)$ in the discrete-time domain can be written as

$V_{P, c}(k)=f_{c, 2}\left(x_{s o c}, \theta\left(x_{s o c}\right), V_{P, c}(k), I_{L}, T_{S}\right)+\Delta V_{P, c}(k)$

$V_{P, c}(k)=f_{c, 2}\left(R_{\text {int }}\left(x_{\text {soc }}\right), \rho\left(x_{\text {soc }}\right), \omega\left(x_{\text {soc }}\right), V_{P, c}(k), I_{L}, T_{S}\right)(10)$ Similarly, the polarization voltage as a function of SoE is expressed as

$V_{P, e}(k)=f_{e, 2}\left(x_{\text {soe }}, \theta\left(x_{\text {soe }}\right), V_{P, e}(k), I_{L}, T_{S}\right)+\Delta V_{P, e}(k)$

$V_{P, e}(k)=f_{e, 2}\left(R_{\text {int }}\left(x_{\text {soe }}\right), \rho\left(x_{\text {soe }}\right), \omega\left(x_{\text {soe }}\right), V_{P, e}(k), I_{L}, T_{S}\right)$

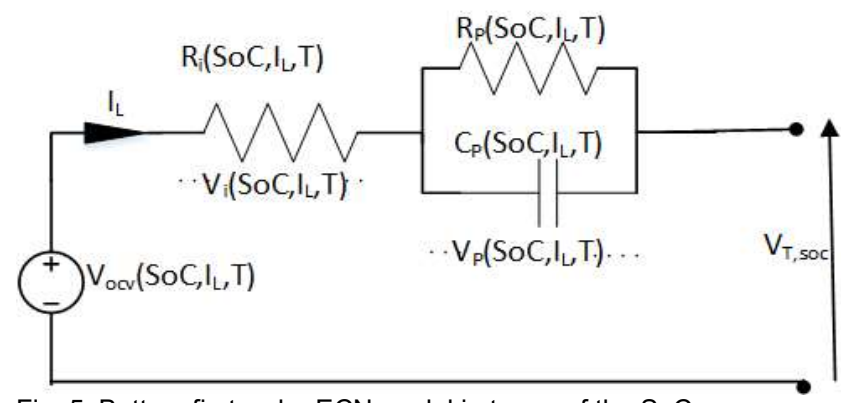

Fig. 5. Battery first-order ECN model in terms of the SoC

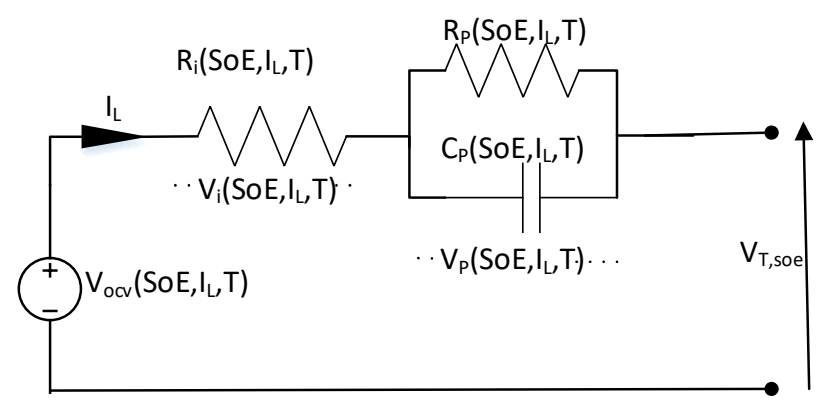

Fig. 6. Battery 1-RC ECN model in terms of the SoE 


\section{Output Voltage Model}

Considering the $1 \mathrm{RC}$ network given in fig 5 as a closed circuit, the output voltage of battery can be written as a function of $V_{o c}($.$) ,$ $V_{P}($.$) , and V_{i}($.$) . Since the open circuit voltage V_{o c}($.$) is a$ function of the state of the battery, the terminal voltage for SoC estimation problem is expressed in terms of the SoC as Similarly, for SoE estimation as, the terminal voltage is expressed as function of SoE

$g_{c}()=.V_{T, c}=V_{o c v}\left(x_{s o c}(k)\right)-V_{P}(k)-V_{i}(k)$

For simplicity, the notation for $\mathrm{I}_{\mathrm{L}}, \mathrm{T}$ is dropped in (31)-(32) and hereafter. Considering voltage sensor noise, the measurement model for SoC as

$y_{c}(k)=g_{c}(k)+v_{s}(k)$

Similarly, the terminal voltage for SoE estimation problem is written as

$y_{e}(k)=g_{e}(k)+v_{s}(k)$

where

$g_{e}\left(x_{e}, \theta_{e}\right)=V_{T, e}=V_{o c v}\left(x_{\text {soe }}(k)\right)-V_{P}(k)-V_{i}(k)$

$v_{S}(k) \sim N\left(0, R_{s}\right)$ is a Gaussian noise. The term $v(k)$ denotes a Gaussian noise with zero mean and known covariance, $R_{c}(k)$.

The SoE estimation could be a redundancy to SoC because the SoE equals SoC when battery open-circuit voltage equals to its terminal voltage [24]. and $\mathrm{SoC}$ is 0 , the battery do not have energy ( $\mathrm{SoE}$ is 0 ) and when SoC is $100 \%$ the battery has maximum energy, i.e. SoE $100 \%$ [13].

\section{Estimation PROBLEM ANd BAyESIAN FILTERING}

\section{A. The State Estimation Problem}

The probabilistic state transition model for SoC estimation is $x_{c}(k) \sim p\left(x_{c}(k) \mid x_{c}(k-1), \theta\left(x_{c, k-1}\right), u_{k}, w_{k}\right)$ where $x_{c}(k) \in \mathbb{R}^{n_{x}}=\left(\begin{array}{c}x_{s o c, k} \\ V_{P, s o c, k}\end{array}\right)=\left(\begin{array}{c}x_{c, 1}(k) \\ x_{c, 2}(k)\end{array}\right)$ denotes the state vector of the battery system, $p\left(x_{c}(k) \mid x_{c}(k-1)\right)$ is the probability density function of the state sequence $\left\{x_{c}(k), k \in \mathbb{N}\right\}$ that represent a state transition model with nonlinear function $f_{c}(k): \mathbb{R}^{n_{x}} \times \mathbb{R}^{n_{\theta}} \times \mathbb{R}^{n_{u}} \times \mathbb{R}^{n_{w}} \rightarrow \mathbb{R}^{n_{x}}$ as

$x_{c}(k)=f_{c}\left(x_{c}(k-1), \theta_{c}(k-1), u(k)\right)+w(k)$,

and $\{w(k), k \in \mathbb{N}\}$ is the independent and identically distributed (i.i.d) process noise. The probabilistic measurement model for SoC estimation is written as

$y_{c k} \sim p\left(y_{c, k} \mid x_{c, k}, \theta\left(x_{c, k-1}\right), u_{k-1}, v_{k}\right)$

where $y_{c}(k) \in \mathbb{R}^{n_{y}}$ is the measurement at time instant $k$, and $p\left(y_{c}(k) \mid x_{c}(k)\right)$ is the probability density function of measurement sequence $\left\{y_{c}(k), k \in \mathbb{N}\right\}$ that represent a measurement model with nonlinear function $g_{\mathrm{c}}(k): \mathbb{R}^{n_{y}} \times$ $\mathbb{R}^{n_{\theta}} \times \mathbb{R}^{n_{u}} \times \mathbb{R}^{n_{v}} \rightarrow \mathbb{R}^{n_{y}}$ as

$y_{c}(k)=g_{c}\left(x_{c}(k), \theta_{c}(k), u(k)\right)+v(k)$,

and $\{v(k), k \in \mathbb{N}\}$ is i.i.d measurement noise. In () and (), $u(k)$ is the inputs such as the load current and temperature, and $\theta_{c}(k)=\left[V_{o c}\left(x_{c, 1}(k)\right), R_{\text {int }}\left(x_{c, 1}(k)\right), \Omega\left(x_{c, 1}(k)\right), \rho\left(x_{c, 1}(k)\right)\right]^{\prime}$.

The $\theta_{c}(k)$ is estimated by the PEM [11]. It is also assume that the initial pdf $p\left(x_{c}(0) \mid y_{c}(0)\right)$, of state vector $x_{c}(k)$ is known though no measurement is made available $y_{c}(0)$, the covariance of processes is given as

$Q=Q_{c}=Q_{e}=\left[\begin{array}{cc}Q_{x} & 0 \\ 0 & Q_{v}\end{array}\right]$ where $Q_{x}$ denotes variance in the states such as SoC and SoE, $Q_{V}$ denotes variance in the polarization voltage. Note that $Q_{V}$ does not have the effect of parameter variation due to temperature hange. Similarly, the covariance of measurement is given as

$R=R_{c}=R_{e}=R_{s}$

where $R_{S}$ is variance of voltage sensor noise.

\section{B. The Traditional Bayesian Filtering Framework}

According to the general Bayesian filtering [13], the posteriori pdf $\hat{x}_{c}(k \mid k)$ of state vector $x_{c}(k)$ from the available measurement $y_{c}(1: k)=\left\{y_{c}(i), i=1, \ldots, k\right\}$ can be evaluated as

$$
p\left(x_{c}(k) \mid y_{c}(1: k)\right)=\frac{p\left(y_{c}(k) \mid x_{c}(k)\right) p\left(x_{c}(k) \mid y_{c}(1: k-1)\right)}{p\left(y_{c}(k) \mid y_{c}(1: k-1)\right)}
$$

where

$p\left(x_{c}(k) \mid y_{c}(1: k-1)\right)=$

$$
\int p\left(x_{c}(k) \mid x_{c}(k-1)\right) p\left(x_{c}(k-1) \mid y_{c}(k-1)\right) d x_{c}(k-1)(24)
$$

is the priori pdf of state vector is also known as prediction state vector, and $p\left(y_{c}(k) \mid x_{c}(k)\right)$ is the likelihood function evaluated by the measurement model with additive noise $v(k) \sim N(0, R)$ as (), and

$$
\begin{aligned}
& p\left(y_{c}(k) \mid y_{c}(1: k-1)\right)= \\
& \quad \int p\left(y_{c}(k) \mid x_{c}(k), \theta_{c}(k-1)\right) p\left(x_{c}(k) \mid y_{c}(1: k-1)\right) d x_{c}(k)
\end{aligned}
$$$$
\text { is a normalized constant. }
$$

Approximating $p\left(x_{c}(k) \mid y_{c}(1: k)\right)$ by Gaussian and linearizing the nonlinear function at local operating point, the conventional extended Kalman filter [\&\&], which is one of the nonlinear Bayesian filters, propagates the priori and posteriori estimated state and error covariance of $x_{c}(k)$ as follow:

$$
\begin{aligned}
& p\left(x_{c}(k-1) \mid y_{c}(1: k-1)\right) \\
& \quad \approx N\left(x_{c}(k-1) ; \hat{x}_{c}(k-1 \mid k-1), P_{c}(k-1 \mid k-1)\right) \\
& p\left(x_{c}(k) \mid y_{c}(1: k-1)\right) \approx N\left(x_{c}(k) ; \hat{x}_{c}(k \mid k-1), P(k \mid k-1)\right) \\
& p\left(x_{c}(k) \mid y_{c}(1: k)\right) \approx N\left(x_{c}(k) ; \hat{x}_{c}(k \mid k), P(k \mid k)\right)
\end{aligned}
$$

The conventional EKF algorithm [9] diverges for both SoC and SoE estimation due to highly nonlinear behavior of $\mathrm{ECN}$ parameters. Using other nonlinear estimators may converges, but we interest to use EKF as it is computationally efficient and easy to implement. Therefore, a novel strategy is introduced for EKF based SoC and SoE estimators of Li-S batteries follow.

\section{Proposed Bayesian State Estimation Strategy}

The typical high- and low-plateau characteristics of Li-S battery state estimation problem dealt by partitioning the ECN parameters into two segments and then evaluating priori state and likelihood accordingly. o smoothly dealt highly nonlinearity of Li-S battery SoC and SoE estimation, the ECN parameters are partitioned as

$$
\theta_{c}(k)=\left\{\begin{array}{l}
\theta_{c, h}(k), 1 \geq x_{s o c}(k) \geq x_{j} \\
\theta_{c, l}(k), x_{j} \geq x_{s o c}(k) \geq 0
\end{array} .\right.
$$

where

$\theta_{c, h}(k)=\left[\begin{array}{llll}V_{o c, h}(.) & R_{\text {int }, h}(.) & \Omega_{h}(.) & \rho_{h}(.)\end{array}\right]^{\prime}$

Is high-plateau fitting models

$\theta_{c, l}(k)=\left[\begin{array}{llll}V_{o c, l}(.) & R_{\text {int }, l}(.) & \Omega_{l}(.) & \rho_{l}(.)\end{array}\right]^{\prime}$

is low-plateau fitting models of ECN parameters as given in the table II. Based on these parameters, the proposed strategy propagates any one of two priori pdfs and likelihood functions as follow: 
$p\left(x_{c}(k) \mid y_{c}(1: k-1)\right)=$

$\begin{cases}p\left(x_{c}(k) \mid y_{c}(1: k-1), \theta_{c, h}(k-1)\right), & 1 \geq x_{s o c}(k) \geq x_{j} \\ p\left(x_{c}(k) \mid y_{c}(1: k-1), \theta_{c, l}(k-1)\right), & x_{j} \geq x_{s o c}(k) \geq 0\end{cases}$

$p\left(y_{c}(k) \mid x_{c}(k)\right)=$

$\begin{cases}p\left(y_{c}(k) \mid x_{c}(1: k-1), \theta_{c, h}(k-1)\right), & 1 \geq x_{s o c}(k) \geq x_{j} \\ p\left(y_{c}(k) \mid x_{c}(1: k-1), \theta_{c, l}(k-1)\right), & x_{j} \geq x_{s o c}(k) \geq 0\end{cases}$

The ECN parameter vector is parameter vector is given as

$\theta_{c}(k)=\left[V_{o c}\left(x_{c, 1}(k)\right), R_{\text {int }}\left(x_{c, 1}(k)\right), \Omega\left(x_{c, 1}(k)\right), \rho\left(x_{c, 1}(k)\right)\right]^{\prime}$

Similarly, the state vector of SoE comprises the two components;

the state-of-energy $x_{\text {soe }}(k)$, and the polarization voltage, $V_{P, \text { soe }}(k)$ which is a function of SoE. The following state vector is used in SoE estimation problem

$$
\begin{gathered}
x(k)=x_{e}(k)=\left(\begin{array}{c}
x_{\text {soe }}(k) \\
V_{P, \text { soe }}(k)
\end{array}\right)=\left(\begin{array}{l}
x_{e, 1}(k) \\
x_{e, 2}(k)
\end{array}\right) \\
\theta_{e}(k)=\left\{\begin{array}{l}
\theta_{e, h}(k), 1 \geq x_{\text {soc }}(k) \geq x_{j} \\
\theta_{e, l}(k), x_{j} \geq x_{\text {soc }}(k) \geq 0
\end{array}\right.
\end{gathered}
$$

where

$\theta_{e, h}(k)=\left[\begin{array}{llll}V_{o c, h}(.) & R_{\text {int }, h}(.) & \Omega_{h}(.) & \rho_{h}(.)\end{array}\right]^{\prime}$

Is high-plateau fitting models

$\theta_{e, l}(k)=\left[\begin{array}{llll}V_{o c, l}(.) & R_{\text {int }, l}(.) & \Omega_{l}(.) & \rho_{l}(.)\end{array}\right]^{\prime}$

is low-plateau fitting models of ECN parameters as given in the table II. Based on these parameters, the proposed strategy propagates any one of two priori pdfs and likelihood functions as follow:

$p\left(x_{e}(k) \mid y_{e}(1: k-1)\right)=$

$\begin{cases}p\left(x_{e}(k) \mid y_{e}(1: k-1), \theta_{e, h}(k-1)\right), & 1 \geq x_{\text {soc }}(k) \geq x_{j} \\ p\left(x_{e}(k) \mid y_{e}(1: k-1), \theta_{e, l}(k-1)\right), & x_{j} \geq x_{s o c}(k) \geq 0\end{cases}$

$p\left(y_{e}(k) \mid x_{e}(k)\right)=$

$\begin{cases}p\left(y_{e}(k) \mid x_{e}(1: k-1), \theta_{e, h}(k-1)\right), & 1 \geq x_{s o c}(k) \geq x_{j} \\ p\left(y_{e}(k) \mid x_{e}(1: k-1), \theta_{e, l}(k-1)\right), & x_{j} \geq x_{s o c}(k) \geq 0\end{cases}$

The last term of right side of (1) and (2) are the Gaussian noise components $w(k) \sim N(0, Q)$ and that represent the uncertainty in process and measurement, respectively, and they are identically independent distribution (i.i.d)

According to (39-40), in high-plateau segment the priori state, approximation of nonlinear functions and likelihood are evaluated. If the $\mathrm{SoC}$ is in the high-plateau region, the proposed EKF based SoC and SoE estimators propagate the priori state vectors $\hat{x}_{c}(k \mid k-1)$ and $\hat{x}_{e}(k \mid k-1)$ of Li-S battery cells as follow:

$$
\begin{aligned}
& {\left[\hat{x}_{c}(k+1 \mid k), \hat{x}_{e}(k+1 \mid k)\right]} \\
& =\hat{f}_{x, h}\left(\hat{x}_{c}(k \mid k), \hat{x}_{e}(k \mid k), \hat{\theta}_{c, h}\left(\hat{x}_{c}(k \mid k)\right), \hat{\theta}_{e, h}\left(\hat{x}_{c}(k \mid k)\right), I_{L}, T_{s}, T\right)(41) \\
& \text { where } \\
& \hat{\theta}_{c, h}\left(\hat{x}_{c}(k \mid k)\right)=\left[\begin{array}{llll}
0 & \hat{R}_{\text {int }, c, h} & \widehat{\omega}_{c, h} & \hat{\rho}_{c, h}
\end{array}\right]^{\prime} \\
& \hat{\theta}_{e, h}\left(\hat{x}_{c}(k \mid k)\right)=\left[\begin{array}{llll}
\hat{V}_{o c, e, h} & \hat{R}_{\text {int }, e, h} & \widehat{\omega}_{e, h} & \hat{\rho}_{e, h}
\end{array}\right]^{\prime} \\
& \text { The following steps are used in (\$\$) } \\
& \hat{R}_{\text {int }, c, h}=\hat{R}_{\text {int }, h, 1} \hat{x}_{c, 1}^{n}(k \mid k)+\hat{R}_{\text {int }, h, 2} \hat{x}_{c, 1}^{n-1}(k \mid k)+\ldots \\
& +\hat{R}_{\text {int }, h, n} \hat{x}_{c, 1}(k \mid k)+\hat{R}_{\text {int }, h, n+1} \\
& \hat{R}_{\text {int }, e, h}=\hat{R}_{i n t, h, 1} \hat{x}_{c, 1}^{n}(k \mid k)+\hat{R}_{\text {int }, h, 2} \hat{x}_{c, 1}^{n-1}(k \mid k)+\cdots \\
& +\hat{R}_{\text {int }, e, h, n} \hat{x}_{c, 1}(k \mid k)+\hat{R}_{\text {int }, c, h, n+1} \\
& \widehat{\omega}_{c, h}=\widehat{\omega}_{c, h, 1} \hat{x}_{c, 1}^{n}(k \mid k)+\widehat{\omega}_{c, h, 2} \hat{x}_{c, 1}^{n-1}(k \mid k)+\cdots+\widehat{\omega}_{c, h, n} \hat{x}_{c, 1}(k \mid k)+ \\
& \widehat{\omega}_{c, h, n+1}
\end{aligned}
$$

$$
\begin{aligned}
& \widehat{\omega}_{e, h}=\widehat{\omega}_{h, 1} \hat{x}_{e, 1}^{n}(k \mid k)+\widehat{\omega}_{h, 2} \hat{x}_{e, 1}^{n-1}(k \mid k)+\cdots+\widehat{\omega}_{h, n} \hat{x}_{e, 1}(k \mid k)+ \\
& \widehat{\omega}_{h, n+1} \\
& \hat{\rho}_{c, h}=\hat{\rho}_{h, 1} \hat{x}_{c, 1}^{n}(k \mid k)+\hat{\rho}_{h, 2} \hat{x}_{c, 1}^{n-1}(k \mid k)+\cdots+\hat{\rho}_{h, n} \hat{x}_{c, 1}(k \mid k)+ \\
& \hat{\rho}_{h, n+1} \\
& \hat{\rho}_{e, h}=\hat{\rho}_{h, 1} \hat{x}_{e, 1}^{n}(k \mid k)+\hat{\rho}_{h, 2} \hat{x}_{e, 1}^{n-1}(k \mid k)+\cdots+\hat{\rho}_{h, n} \hat{x}_{e, 1}(k \mid k)+ \\
& \hat{\rho}_{h, n+1} \\
& \hat{x}_{c}(k+1 \mid k)=\left[\hat{x}_{c, 1}(k \mid k)-T_{s} \frac{\left|I_{L}\right|}{C_{B}} ; \hat{x}_{c, 2}(k \mid k)-T_{s} \widehat{\omega}_{c, h} \hat{x}_{c, 2}(k \mid k)\right. \\
& \left.+T_{s} \hat{\rho}_{c, h} \hat{R}_{\text {int }, c, h} \widehat{\omega}_{c, h} I_{L}\right] \\
& \hat{V}_{o c, e, h}=\widehat{V}_{o c, h, 1} \hat{x}_{e, 1}^{n}(k \mid k)+\widehat{V}_{o c, h, 2} \hat{x}_{e, 1}^{n-1}(k \mid k)+\cdots+ \\
& \widehat{V}_{o c, h, n} \hat{x}_{e, 1}(k \mid k)+\hat{V}_{o c, h, n+1} \\
& \hat{x}_{e}(k+1 \mid k)=\left[\hat{x}_{e, 1}(k \mid k)-T_{S} \frac{I_{L} \widehat{V}_{o c, e, h}}{E_{B}} ; \hat{x}_{e, 2}(k \mid k)-\right. \\
& T_{s} \widehat{\omega}_{e, h} \hat{x}_{e, 2}(k \mid k)+T_{s} \hat{\rho}_{e, h} \hat{R}_{i n t, e, h} \widehat{\omega}_{e, h} I_{L}
\end{aligned}
$$

The $F_{c}(k)$ and $F_{e}(k)$ are Jacobian for SoC and SoE respectively.

The $\hat{V}_{c}(k+1)$ and $\hat{V}_{e}(k+1)$ are likelihood of SoC and SoE estimation, respectively, and the function $g_{h}\left(x_{c}(k), \theta_{c}(k), u(k)\right)$ returns them by the following steps

$\tilde{\theta}_{c, h}\left(\hat{x}_{c}(k+1 \mid k)\right)=\left[\begin{array}{llll}\tilde{V}_{o c, c, h} & \widetilde{R}_{i n t, c, h} & 0 & \tilde{\rho}_{c, h}\end{array}\right]^{\prime}$

$\tilde{\theta}_{e, h}\left(\hat{x}_{c}(k+1 \mid k)\right)=\left[\begin{array}{llll}\tilde{V}_{o c, e, h} & \widetilde{R}_{\text {int }, e, h} & 0 & \tilde{\rho}_{e, h}\end{array}\right]^{\prime}$

$$
\left[\hat{V}_{c}(k+1), \widehat{V}_{e}(k+1)\right]
$$$$
=g_{h}\left(\hat{x}_{c}(k+\mid k), \hat{x}_{e}(k+\mid k), \tilde{\theta}_{c, h}, \tilde{\theta}_{e, h}, I_{L}, T_{s}, T\right)
$$

$\tilde{V}_{o c, c}\left(\hat{x}_{c, 1}(k+\mid k)\right)=V_{o c, h, 1} \hat{x}_{c, 1}^{n}(k+1 \mid k)+V_{o c, h, 2} \hat{x}_{c, 1}^{n-1}(k+1 \mid k)$

$$
+\cdots+V_{o c, h, n} \hat{x}_{c, 1}(k+1 \mid k)+V_{o c, h, n+1}
$$

$\tilde{V}_{o c, e}\left(\hat{x}_{e, 1}(k+\mid k)\right)=V_{o c, h, 1} \hat{x}_{e, 1}^{n}(k+1 \mid k)+V_{o c, h, 2} \hat{x}_{e, 1}^{n-1}(k+1 \mid k)$ $+\cdots+V_{o c, h, n} \hat{x}_{e, 1}(k+1 \mid k)+V_{o c, h, n+1}$

$\tilde{R}_{\text {int }, c}\left(\hat{x}_{c, 1}(k+\mid k)\right)=R_{\text {int }, h, 1} \hat{x}_{c, 1}^{n}(k+1 \mid k)$ $+R_{\text {int }, h, 2} \hat{x}_{c, 1}^{n-1}(k+1 \mid k)+\cdots$

$+R_{\text {int }, h, n} \hat{x}_{c, 1}(k+1 \mid k)+R_{\text {int }, h, n+1}$

$\tilde{R}_{\text {int }, e}\left(\hat{x}_{e, 1}(k+\mid k)\right)=R_{\text {int }, h, 1} \hat{x}_{e, 1}^{n}(k+1 \mid k)$ $+R_{\text {int }, h, 2} \hat{x}_{e, 1}^{n-1}(k+1 \mid k)+$

$\ldots+R_{\text {int }, h, n} \hat{x}_{e, 1}(k+1 \mid k)+R_{\text {int }, h, n+1}$

$\tilde{\rho}_{c, h}\left(\hat{x}_{c, 1}(k+\mid k)\right)=\rho_{h, 1} \hat{x}_{c, 1}^{n}(k+1 \mid k)$ $+\rho_{h, 2} \hat{x}_{c, 1}^{n-1}(k+1 \mid k)+\cdots$

$+\rho_{h, n} \hat{x}_{c, 1}(k+1 \mid k)+\rho_{h, n+1}$

$$
\begin{aligned}
\tilde{\rho}_{e, h}\left(\hat{x}_{e, 1}(k+\mid k)\right) & =\rho_{h, 1} \hat{x}_{e, 1}^{n}(k+1 \mid k) \\
& +\rho_{h, 2} \hat{x}_{e, 1}^{n-1}(k+1 \mid k)+ \\
\ldots+ & \rho_{h, n} \hat{x}_{e, 1}(k+1 \mid k)+\rho_{h, n+1}
\end{aligned}
$$

$\hat{V}_{t, c}(k+1)=\tilde{V}_{o c, c}-\hat{x}_{c, 2}(k+\mid k)-\left(1-\tilde{\rho}_{c, h}().\right) \tilde{R}_{\text {int }, c, h} I_{L}$

$\hat{V}_{t, e}(k+1)=\tilde{V}_{o c, e}-\hat{x}_{e, 2}(k+\mid k)-\left(1-\tilde{\rho}_{e, h}().\right) \tilde{R}_{\text {int }, e, h} I_{L}$

Using the above priori, the nonlinear measurement functions are approximated. In EKF the Jacobians $H_{c}$ and $H_{e}$ are nonlinear approximation of voltage for $\mathrm{SoC}$ and $\mathrm{SoE}$ state estimation respectively. These priori state and likelihood propagate as same as strategy given in fig 6 of [11], which is a novel strategy.

$H_{c}=\left[\begin{array}{ll}\frac{\partial g_{c, h}(.)}{\partial x_{c, 1}} & \frac{\partial g_{c, h}(.)}{\partial x_{c, 2}}\end{array}\right]_{\mid x(k)=\hat{x}_{c}(k+1 \mid k)}$
$H_{e}=\left[\begin{array}{ll}\frac{\partial g_{e, h}(.)}{\partial x_{e, 1}} & \frac{\partial g_{e, h}(.)}{\partial x_{e, 2}}\end{array}\right]_{\mid x(k)=\hat{x}_{e}(k+1 \mid k)}$

Like high-plateau, the proposed strategy evaluates the priori state, approximation of system function, likelihood and approximation of nonlinear measurement for $\mathrm{SoC}$ and $\mathrm{SOE}$ estimation in the low-plateau region, as follow:

$\left[\hat{x}_{c}(k+1 \mid k), \hat{x}_{e}(k+1 \mid k)\right]$

$=\hat{f}_{x, l}\left(\hat{x}_{c}(k \mid k), \hat{x}_{e}(k \mid k), \hat{\theta}_{c, l}\left(\hat{x}_{c}(k \mid k)\right), \hat{\theta}_{e, l}\left(\hat{x}_{c}(k \mid k)\right), I_{L}, T_{s}, T\right)$ where 


$$
\begin{aligned}
& \hat{\theta}_{c, l}\left(\hat{x}_{c}(k \mid k)\right)=\left[\begin{array}{llll}
0 & \hat{R}_{\text {int }, c, l} & \widehat{\omega}_{c, l} & \hat{\rho}_{c, l}
\end{array}\right]^{\prime} \\
& \hat{\theta}_{e, l}\left(\hat{x}_{c}(k \mid k)\right)=\left[\begin{array}{llll}
\hat{V}_{o c, e, l} & \hat{R}_{\text {int }, e, l} & \widehat{\omega}_{e, l} & \hat{\rho}_{e, l}
\end{array}\right]^{\prime} \\
& \text { The following steps are used in (\$\$) } \\
& \hat{R}_{i n t, c, l}=\hat{R}_{i n t, l, 1} \hat{x}_{c, 1}^{n}(k \mid k)+\hat{R}_{i n t, l, 2} \hat{x}_{c, 1}^{n-1}(k \mid k)+\ldots \\
& +\hat{R}_{i n t, l, n} \hat{x}_{c, 1}(k \mid k)+\hat{R}_{i n t, l, n+1} \\
& \hat{R}_{\text {int }, e, l}=\hat{R}_{\text {int }, l, 1} \hat{x}_{c, 1}^{n}(k \mid k)+\hat{R}_{\text {int }, l, 2} \hat{x}_{c, 1}^{n-1}(k \mid k)+\cdots \\
& +\hat{R}_{\text {int }, e, l, n} \hat{x}_{c, 1}(k \mid k)+\hat{R}_{\text {int }, c, l, n+1} \\
& \widehat{\omega}_{c, l}=\widehat{\omega}_{l, 1} \hat{x}_{c, 1}^{n}(k \mid k)+\widehat{\omega}_{l, 2} \hat{x}_{c, 1}^{n-1}(k \mid k)+\cdots+\widehat{\omega}_{l, n} \hat{x}_{c, 1}(k \mid k)+ \\
& \widehat{\omega}_{l, n+1} \\
& \widehat{\omega}_{e, l}=\widehat{\omega}_{l, 1} \hat{x}_{e, 1}^{n}(k \mid k)+\widehat{\omega}_{l, 2} \hat{x}_{e, 1}^{n-1}(k \mid k)+\cdots+\widehat{\omega}_{l, n} \hat{x}_{e, 1}(k \mid k)+ \\
& \widehat{\omega}_{l, n+1} \\
& \hat{\rho}_{c, l}=\hat{\rho}_{l, 1} \hat{x}_{c, 1}^{n}(k \mid k)+\hat{\rho}_{l, 2} \hat{x}_{c, 1}^{n-1}(k \mid k)+\cdots+\hat{\rho}_{l, n} \hat{x}_{c, 1}(k \mid k)+ \\
& \hat{\rho}_{l, n+1} \\
& \hat{\rho}_{e, l}=\hat{\rho}_{l, 1} \hat{x}_{e, 1}^{n}(k \mid k)+\hat{\rho}_{l, 2} \hat{x}_{e, 1}^{n-1}(k \mid k)+\cdots+\hat{\rho}_{l, n} \hat{x}_{e, 1}(k \mid k)+ \\
& \hat{\rho}_{l, n+1} \\
& \hat{x}_{c}(k+1 \mid k)=\left[\hat{x}_{c, 1}(k \mid k)-T_{s} \frac{\left|I_{L}\right|}{C_{B}} ; \hat{x}_{c, 2}(k \mid k)-T_{s} \widehat{\omega}_{c, l} \hat{x}_{c, 2}(k \mid k)\right. \\
& \left.+T_{s} \hat{\rho}_{c, l} \hat{R}_{i n t, c, l} \widehat{\omega}_{c, l} I_{L}\right] \\
& \hat{V}_{o c, e, l}=\widehat{V}_{o c, l, 1} \hat{x}_{e, 1}^{n}(k \mid k)+\widehat{V}_{o c, l, 2} \hat{x}_{e, 1}^{n-1}(k \mid k)+\cdots+ \\
& \hat{V}_{o c, l, n} \hat{x}_{e, 1}(k \mid k)+\hat{V}_{o c, l, n+1} \\
& \hat{x}_{e}(k+1 \mid k)=\left[\hat{x}_{e, 1}(k \mid k)-T_{s} \frac{I_{L} \widehat{V}_{o c, e, l}}{E_{B}} ; \hat{x}_{e, 2}(k \mid k)-\right. \\
& T_{s} \widehat{\omega}_{e, l} \hat{x}_{e, 2}(k \mid k)+T_{s} \hat{\rho}_{e, l} \hat{R}_{i n t, e, l} \widehat{\omega}_{e, l} I_{L}
\end{aligned}
$$

Using the low plateau priori estimate SoC and SoE, the Jacobian $F_{c}(k)$ and $F_{e}(k)$, of nonlinear system equations are evaluated. These Jacobians are used to evaluate the likelihood.

Let the $\widehat{V}_{c}(k+1)$ and $\hat{V}_{e}(k+1)$ are likelihood of SoC and SoE estimation, respectively. The function $g_{l}\left(x_{c}(k), \theta_{c}(k), u(k)\right)$ returns them by the following steps

$$
\begin{aligned}
& \tilde{\theta}_{c, l}\left(\hat{x}_{c}(k+1 \mid k)\right)=\left[\begin{array}{llll}
\tilde{V}_{o c, c, l} & \widetilde{R}_{i n t, c, l} & 0 & \tilde{\rho}_{c, l}
\end{array}\right]^{\prime} \\
& \tilde{\theta}_{e, l}\left(\hat{x}_{c}(k+1 \mid k)\right)=\left[\begin{array}{llll}
\tilde{V}_{o c, e, l} & \widetilde{R}_{\text {int }, e, l} & 0 & \tilde{\rho}_{e, l}
\end{array}\right]^{\prime} \\
& {\left[\hat{V}_{c}(k+1), \hat{V}_{e}(k+1)\right]} \\
& =g_{l}\left(\hat{x}_{c}(k+\mid k), \hat{x}_{e}(k+\mid k), \tilde{\theta}_{c, l}, \tilde{\theta}_{l, h}, I_{L}, T_{s}, T\right) \\
& \tilde{V}_{o c, c, l}\left(\hat{x}_{c, 1}(k+\mid k)\right)=V_{o c, l, 1} \hat{x}_{c, 1}^{n}(k+1 \mid k)+V_{o c, l, 2} \hat{x}_{c, 1}^{n-1}(k+1 \\
& +\cdots+V_{o c, l, n} \hat{x}_{c, 1}(k+1 \mid k)+V_{o c, l, n+1} \\
& \tilde{V}_{o c, e, l}\left(\hat{x}_{e, 1}(k+\mid k)\right)=V_{o c, l, 1} \hat{x}_{e, 1}^{n}(k+1 \mid k)+V_{o c, l, 2} \hat{x}_{e, 1}^{n-1}(k+1 \mid k) \\
& +\cdots+V_{o c, l, n} \hat{x}_{e, 1}(k+1 \mid k)+V_{o c, l, n+1} \\
& \tilde{R}_{\text {int }, c, l}\left(\hat{x}_{c, 1}(k+\mid k)\right)=R_{\text {int }, l, 1} \hat{x}_{c, 1}^{n}(k+1 \mid k) \\
& +R_{\text {int }, l, 2} \hat{x}_{c, 1}^{n-1}(k+1 \mid k)+\cdots \\
& +R_{\text {int }, l, n} \hat{x}_{c, 1}(k+1 \mid k)+R_{\text {int }, l, n+1} \\
& \tilde{R}_{\text {int }, e, l}\left(\hat{x}_{e, 1}(k+\mid k)\right)=R_{\text {int }, l, 1} \hat{x}_{e, 1}^{n}(k+1 \mid k) \\
& +R_{\text {int }, l, 2} \hat{x}_{e, 1}^{n-1}(k+1 \mid k)+ \\
& \ldots+R_{\text {int }, l, n} \hat{x}_{e, 1}(k+1 \mid k)+R_{\text {int }, l, n+1} \\
& \tilde{\rho}_{c, l}\left(\hat{x}_{c, 1}(k+\mid k)\right)=\rho_{l, 1} \hat{x}_{c, 1}^{n}(k+1 \mid k) \\
& +\rho_{l, 2} \hat{x}_{c, 1}^{n-1}(k+1 \mid k)+\cdots \\
& +\rho_{l, n} \hat{x}_{c, 1}(k+1 \mid k)+\rho_{l, n+1} \\
& \tilde{\rho}_{e, l}\left(\hat{x}_{e, 1}(k+\mid k)\right)=\rho_{l, 1} \hat{x}_{e, 1}^{n}(k+1 \mid k) \\
& +\rho_{l, 2} \hat{x}_{e, 1}^{n-1}(k+1 \mid k)+ \\
& \ldots+\rho_{l, n} \hat{x}_{e, 1}(k+1 \mid k)+\rho_{l, n+1}
\end{aligned}
$$

The approximation of nonlinear measurement functions of SoC and SoE estimators are evaluated as

$H_{c}=\left[\begin{array}{ll}\frac{\partial g_{c, l}(.)}{\partial x_{c, 1}} & \frac{\partial g_{c, l}(\cdot)}{\partial x_{c, 2}}\end{array}\right]_{\mid x(k)=\hat{x}_{c}(k+1 \mid k)}$

$$
H_{e}=\left[\begin{array}{ll}
\frac{\partial g_{e, l}(.)}{\partial x_{e, 1}} & \frac{\partial g_{e, l}(.)}{\partial x_{e, 2}}
\end{array}\right]_{\mid x(k)=\hat{x}_{e}(k+1 \mid k)}
$$

As (89) is different from (64), the proposed Bayesian framework evaluates the likelihood differently than (25). This is likelihood partitioned EKF for SoC estimation and it is due to (32) and (33). Similarly (19) is different from (65), this means the likelihood partitioned in the SoE estimation as well because of (39) and (40). In this same way, the Jacobians for nonlinear system equations. The proposed strategy propagates the priori and posteriori estimation of Li-S battery's SoC and SoE, as fig 6 of [11]. The proposed approach works well for given experiment data as shown in the next section.

\section{DEMONSTRATION USING EXPERIMENTAL TESt DATA}

This section presents the validated results of the proposed EKF based SoC and SoE estimators given in section III using experimental test data given in section II.

For both SoC and SoE estimation, the EKF sets the following initial conditions. The initial estimated error covariance is set to $\mathrm{P}_{\mathrm{c}}(0 \mid 0)=\mathrm{P}_{\mathrm{e}}(0 \mid 0)=\mathrm{I}_{2 \times 2}$. Two different initial estimated state conditions are considered to evaluate the performance of the proposed EKF under two different typical problems. For first case, the initial state vector is set to $\hat{x}_{c}(0 \mid 0)=\hat{x}_{e}(0 \mid 0)=[1,0]^{\prime}$. This means the EKF has no error in the initial SoC and SoE. For second case, $\hat{\mathrm{x}}_{\mathrm{c}}(0 \mid 0)=\hat{\mathrm{x}}_{\mathrm{e}}(0 \mid 0)=[0.6,0]^{\prime}$. This means there is 40 percent error in the initial states. As $x_{j}=0.7$ is used in (32)(33) and (39)-(40) for partition the likelihood of Bayesian method, validation by two different initial conditions, ensures the reliability of the proposed method. Former case is a straightforward, the algorithm recursively propagates state and estimate error covariance of high plateau to low-plateau state of the Li-S batteries. Later case is a nontrivial problem as actual state is high-plateau, but the initial estimated state is in low-plateau. The validation of proposed method under second initial condition ensures the reliability of the algorithm.

Two temperature conditions such as $20^{\circ} \mathrm{C}$ and $30^{\circ} \mathrm{C}$ are considered. Table II and III show parameters of tested Li-S cell at $20^{\circ} \mathrm{C}$ and $30^{\circ} \mathrm{C}$, respectively. These parameters were obtained by $\mathrm{PEM}$ as $[8,11]$.

\section{a. Results at $20^{\circ} \mathrm{C}$}

For simulate the $\mathrm{SoC}$ and $\mathrm{SoE}$ estimation of cell $20^{\circ} \mathrm{C}$, the proposed EKF uses the fitted ECN parameters of Li-S cell at $20^{\circ} \mathrm{C}$ given in the Table III.

Fig. 7 compares the estimated state of Li-S cell at $20^{\circ} \mathrm{C}$ to corresponding theoretical or true state. For instance, Fig 7(a) compares the estimated $\mathrm{SoC}$ in $\mathrm{Ah}$ to that of true $\mathrm{SoC}$ of cell at $20^{\circ} \mathrm{C}$. It is observed that the considered $\mathrm{Li}-\mathrm{S}$ cell has $2.72 \mathrm{Ah}$ when it was fully charged. Fig.7(b) shows the normalized SoC, which has no unit. In these, the black dotted-line indicated true SoC, whereas the red-dotted line and blue-dashed line denote estimated $\mathrm{SoC}$ when initial SoC is 1 and 0.6 , respectively. In the case of known initial SoC case, the performance of EKF is straight forward, the estimated $\mathrm{SoC}$ by the $\mathrm{EKF}$ immediately follows the true SoC. When the EKF starts with initial SoC is 0.6, the estimated SoC takes couple of minutes to reach its true SoC. It is interesting to note here that the proposed method starts converging from next second. This means that proposed LPB method of EKF when initial state is known and initial state is 
unknown. The accuracy in the SoC estimation is very good, less than five percent error between actual SoC and estimated SoC.

TABLE II

THE ECN PARAMETER FITTING MODEL OF A-CELL AT $20^{\circ} \mathrm{C}$

\begin{tabular}{|c|c|c|c|c|}
\hline Plateau & $\begin{array}{l}V_{o c} \\
(\mathrm{~V})\end{array}$ & $\begin{array}{l}R_{\text {int }} \\
(\Omega)\end{array}$ & $\begin{array}{c}\rho \\
\text { (no unit) }\end{array}$ & $\begin{array}{r}\omega \\
(\mathrm{rad} / \mathrm{s})\end{array}$ \\
\hline High & $\begin{array}{l}\quad\left[V_{o c, h}\right]^{\prime}= \\
{[108.1} \\
-361.13 \\
444.73 \\
-238.18 \\
47.03 \\
1.88]\end{array}$ & $\begin{array}{l}\quad\left[R_{\text {int }, h}\right]^{\prime}= \\
{[1.07} \\
-2.445 \\
1.457]\end{array}$ & $\begin{array}{c}\quad\left[\rho_{h}\right]^{\prime}= \\
{[-0.0451} \\
0.4892]\end{array}$ & $\begin{array}{c}{\left[\omega_{h}\right]^{\prime}=} \\
{[0.415} \\
-0.7441 \\
0.3469]\end{array}$ \\
\hline low & $\begin{array}{l}\quad\left[V_{o c, l}\right]^{\prime}= \\
{[-752.62} \\
2085.66 \\
-2392.87 \\
1466.98 \\
-517.42 \\
105.21 \\
-11.69 \\
0.62 \\
2.1]\end{array}$ & $\begin{array}{l}{\left[R_{\text {int }, l}\right]^{\prime}=} \\
{[3.792} \\
-8.212 \\
6.428 \\
-1.56 \\
0.07346 \\
0.1458]\end{array}$ & $\begin{array}{c}{\left[\rho_{l}\right]^{\prime}=} \\
{[0.5087} \\
-0.6572 \\
0.6815]\end{array}$ & $\begin{array}{l}\quad\left[\omega_{l}\right]^{\prime}= \\
{[6.8270} \\
-7.4220 \\
1.6850 \\
0.1407 \\
-0.1544 \\
0.1441]\end{array}$ \\
\hline
\end{tabular}
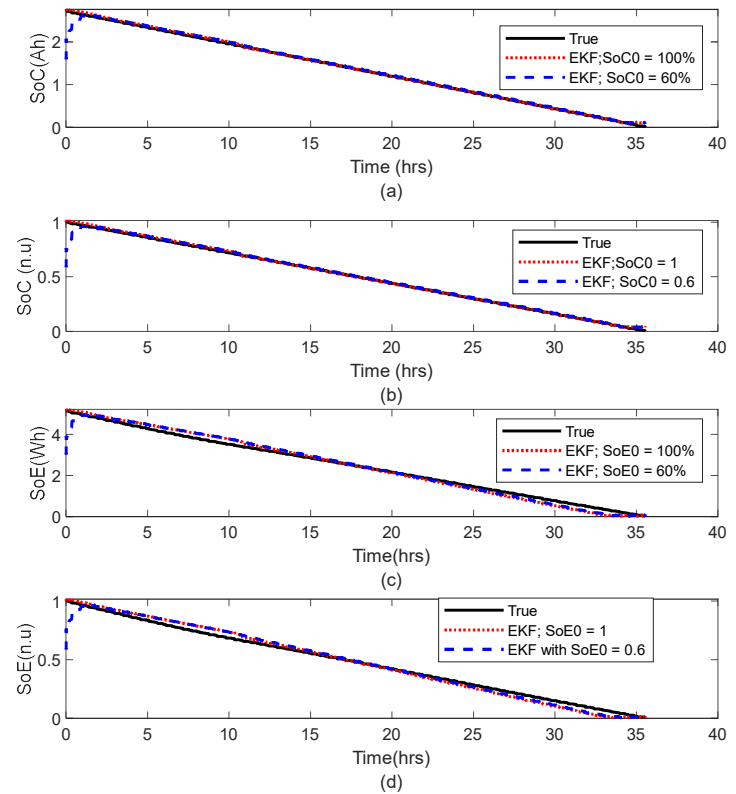

Fig. 7. Estimated vs True states of Li-S cell at $20^{\circ} \mathrm{C}$ under two different state; (a) SoC in Ah (b) SoC normalized (c) SoE in Wh (d) SoEnormalized.

Like SoC estimation, the SoE estimation by the proposed EKF is accurate as shown in the Fig 7(c) and 7(d). In both these, the red-dotted line and blue-dashed line denote the estimated SoE of Li-S type A cell at $20^{\circ} \mathrm{C}$ when initial state is 1 and 0.6 , respectively. Fig8(c) compares the estimated $\mathrm{SoE}$ in Wh to that of true SoE. It is an evident that when considered Li-S type A is fully charged, it has $5.1 \mathrm{Wh}$ energy, and that estimated accurately by the EKF as shown in Fig 7(c). The estimation of SoE in Wh can be directly used for energy/power optimization [16] of Li-S battery powered air vehicles. In Fig 7(d) the normalized SoE estimation is compared. The performance is good as the accuracy of SoE estimation is good, less than five percent error. While comparing SoE to the SoE estimation, the SoC estimation is more accurate. Particularly, at the end of discharge and transition of high-and low-plateau regions, the SoE estimation has slight deviation from the actual SoE. This may be the impact of voltage in (3a)-3(b). Though there is slight difference between SoC and $\mathrm{SoE}$, in overall, the proposed EKF performs well for both SoC and SoE estimation of experimented Li-S cell at $20^{\circ} \mathrm{C}$

\section{b. Results at $30^{\circ} \mathrm{C}$.}

When temperature of $\mathrm{Li}-\mathrm{S}$ cell is $30^{\circ} \mathrm{C}$, the proposed EKF uses the ECN parametrization of given in Table III. Using pulse test data acquired at temperature $30^{\circ} \mathrm{C}$ given in Fig 2, the PEM based ECN parameterization is used here. The simulation results of the novel framework for SoC and $\mathrm{SoE}$ estimation of experimented cell at $30^{\circ} \mathrm{C}$ are observed.

TABLE III

THE ECN PARAMETER FITTING MODEL OF A-CELL AT $30^{\circ} \mathrm{C}$

\begin{tabular}{|c|c|c|c|c|}
\hline Plateau & $\begin{array}{l}V_{o c} \\
(\mathrm{~V})\end{array}$ & $\begin{array}{c}R_{\text {int }} \\
(\Omega)\end{array}$ & $\begin{array}{c}\rho \\
\text { (no unit) }\end{array}$ & $(\mathrm{rad} / \mathrm{s})^{\omega}$ \\
\hline High & $\begin{array}{l}\quad\left[V_{o c, h}\right]^{\prime}= \\
{[72.9503} \\
-259.6135 \\
341.4807 \\
-195.5125 \\
43.1183]\end{array}$ & $\begin{array}{l}\quad\left[R_{\text {int }, h}\right]^{\prime}= \\
{[2.001} \\
-3.759 \\
1.853]\end{array}$ & $\begin{array}{c}\quad\left[\rho_{h}\right]^{\prime}= \\
{[-0.1295} \\
0.5687]\end{array}$ & $\begin{array}{l}\quad\left[\omega_{h}\right]^{\prime}= \\
{[1.0395} \\
-0.7603]\end{array}$ \\
\hline low & $\begin{array}{l}\quad\left[V_{o c, l}\right]^{\prime}= \\
{[-31.2310} \\
69.3765 \\
-60.1029 \\
25.6103 \\
-5.6128 \\
0.5928 \\
2.0974]\end{array}$ & $\begin{array}{l}\quad\left[R_{\text {int,l }}\right]^{\prime}= \\
{[173.7228} \\
-515.0199 \\
616.6895 \\
-381.7758 \\
129.5985 \\
-23.1084 \\
1.6510 \\
0.1450]\end{array}$ & $\begin{array}{l}\quad\left[\rho_{l}\right]^{\prime}= \\
{[-2.1590} \\
2.2718 \\
-23.1084 \\
1.6510]\end{array}$ & $\begin{array}{l}\quad\left[\omega_{l}\right]^{\prime}= \\
{[-1.3532} \\
2.0927 \\
-1.0609 \\
0.1907]\end{array}$ \\
\hline
\end{tabular}

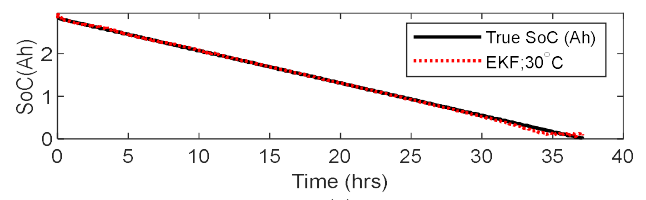

(a)
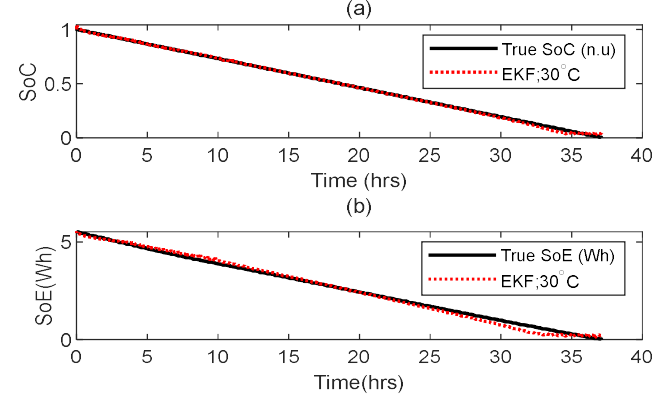

(c)

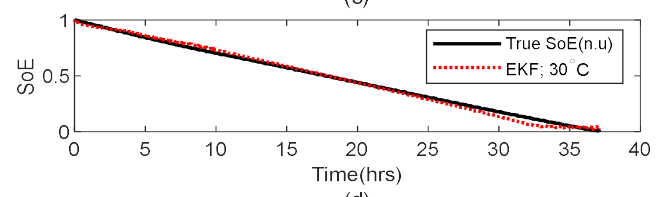

(d)

Fig. 8. Estimated vs True states of Li-S cell at $30^{\circ} \mathrm{C}$; (a) SoC in Ah (b) SoC normalized (c) SoE in Wh (d) SoE normalized.

Fig 8 illustrates a comparison between the $\mathrm{SoC}$ and $\mathrm{SoE}$ estimation and their corresponding true state of given cell at $30^{\circ} \mathrm{C}$. 
The results show that the EKF's perform well at cell at $30^{\circ} \mathrm{C}$ temperature condition. In all subplots of fig 8 , the red-dotted line denotes the estimate state and black-solid line denotes the true state. For instance, the red-dotted line and black-dotted line of Fig 8(a) represent the estimated $\mathrm{SoC}$ in $\mathrm{Ah}$ and true $\mathrm{SoC}$, respectively, in Ah of type A cell at $30^{\circ} \mathrm{C}$. From the beginning of discharge to depletion state of cell, the estimated state by EKF closely follows the true state. The similar trends follow in normalization of SoC as well. Fig 8(b) compares SoC normalised to its normalised true SoC. Both Fig 8(a) and Fig 8(b) show that the estimated SoC by the EKF is almost superimposed the true SoC from the beginning to end of discharge. Similar trend is followed by SoE estimation as well. For instance, Fig 8(c) shows that the estimated SoE in Wh is very close to that true SoE. Similarly, Fig $8(d)$ shows the normalized SoE estimation is accurate as close to the true SoE. The results are clear evidence that the performance of proposed EKF for both SoC and SoE estimation is promising as their accuracy is good.

The accuracy of both SoC and SoE estimation by proposed EKF evaluated in terms of RMSE error and absolute error. The degree of observability [11] analysis is used for RMSE study. In addition, the absolute and squared error between estimated state and its true state are evaluated. As algorithm recalibrated here for improve the smoothness and accuracy, the error is much lower than the fig 12 of [11]. The error plots are not presented here due to page limitation. However, the proposed EKF's accuracy for SoC and SoE estimation of given cell at both temperature conditions is numerically evaluated, and it is about 97 percent.

\section{CONCLUSIONS AND FUTURE WORKS}

A novel likelihood-partitioned Bayesian filter has been developed for estimate the SoC and SoE of lithium-sulfur battery cells. The battery cells are modelled as ECN models, and its parameters are estimated using a prediction error minimization (PEM) method based estimator. the proposed Bayesian filtering strategy is implemented on a simple nonlinear Bayesian filter namely extended Kalman filter (EKF). The proposed EKF strategy has been validated with experimental test data of a particular type of Li-S battery cell. The EKF is calibrated by mixed-C-rate pulse test data at each temperature points. The performance of proposed method for estimating both $\mathrm{SoC}$ and SoE of both cell types is quite acceptable, estimation accuracy is more than 95 percent for given test data under two different temperature and initial conditions. As this novel filter based SoC and SoE estimation does not use additional functions or training sets, it is computationally efficient for coping with the highly nonlinear and complex behaviors of Li-S batteries. By exploring the definitions SoC and SoE in terms of their unit, and their estimation of both Li-S cell series the physical significant difference between the estimated $\mathrm{SoC}$ in $\mathrm{Ah}$ and $\mathrm{SoE}$ in $\mathrm{Wh}$ at different point of times has been studied. The estimated SoC in $\mathrm{Ah}$ and $\mathrm{SoE}$ in Wh can be directly used to the optimization by closed loop energy/or power management control system of electric vehicles. Future research includes to apply the proposed strategy to other applications such as road vehicles, and to explore the robustness against different uncertainties.

\section{ACKNOWLEDGMENT}

The authors thank the Aerospace Technology Institute (ATI) and Innovate UK who funded this work under grants TS/P003818/1 and TS/R013780/1, and European commission gran 814471 . Data used for this research can is subject to an embargo, but will be published at https://doi.org/10.17862/cranfield.rd.14519760.v1

\section{REFERENCES}

[1] Bruce, P., Freunberger, S., Hardwick, L. et al. $\mathrm{Li}-\mathrm{O}_{2}$ and $\mathrm{Li}-\mathrm{S}$ batteries with high energy storage. Nature Mater 11, 19-29 (2012). https://doi.org/10.1038/nmat3191.

[2] Zhao, C., Xu, GL., Yu, Z. et al. A high-energy and long-cycling lithiumsulfur pouch cell via a macroporous catalytic cathode with double-end binding sites. Nat. Nanotechnol. (2020). https://doi.org/10.1038/s41565020-00797-w.

[3] F. Ho Suk Ryu, Zaiping Guo, Hyo Jun Ahn, Gyu Bong Cho, Huakun Liu, Investigation of discharge reaction mechanism of lithium|liquid electrolyte|sulfur battery, Journal of Power Sources, Vol. 189, Issue 2, pp: 1179-1183, 2009. https://doi.org/10.1016/j.jpowsour.2008.12.073.

[4] D. Stroe, V. Knap, M. Swierczynski and E. Schaltz, "Electrochemical Impedance Spectroscopy-Based Electric Circuit Modeling of LithiumSulfur Batteries During a Discharging State," in IEEE Transactions on Industry Applications, vol. 55, no. 1, pp. 631-637, Jan.-Feb. 2019, doi: 10.1109/TIA.2018.2864160.

[5] N. Shateri, Z. Shi, D. J. Auger and A. Fotouhi, "Lithium-Sulfur Cell State of Charge Estimation Using a Classification Technique," in IEEE Transactions on Vehicular Technology. 2020 doi: 10.1109/TVT.2020.3045213.

[6] K. Propp, D. J. Auger, A. Fotouhi, S. Longo, V. Knap, "Kalman-variant estimators for state of charge in Lithium-sulfur batteries," Journal of Power Sources, vol. 343, pp. 254-267, March 2017.

[7] F. A. Fotouhi, D. J. Auger, K. Propp and S. Longo, "Lithium-sulfur battery state-of-charge observability analysis and estimation," IEEE Transactions on Power Electronics, vol.33, no.7, pp. 5847-5859, 2018.

[8] K. Propp, M. Marinescu, D. J. Auger, L. O'Neill, A. Fotouhi, K. Somasundaram, G. J. Offer, G. Minton, S. Longo, M. Wild and V. Knap, "Multi-temperature state-dependent equivalent circuit discharge model for lithium-sulfur batteries," Journal of Power Sources, vol.328, pp.289-299. Oct 2016. Dataset/s: 10.17862/cranfield.rd.c.3292031

[9] K. Propp, D. J. Auger, A. Fotouhi, M. Marinescu, V. Knap, and S. Longo, "Improved State of Charge Estimation for Lithium-Sulfur Batteries," Journal of Energy Storage, 2019, vol. 26, pp. 100943, 2019.

[10] S. Leutenegger, "Unmanned Solar Airplanes: design and algorithms for efficient and robust autonomous operation," Doctor of Sciences Dissertation, ETH Zurich, 2014.

[11] Srinivasan Munisamy, Daniel Auger, Abbas Fotouhi, and Bob Hawkes, "State of energy estimation in electric propulsion systems with Lithiumsulfur batteries," in IET the $10^{\text {th }}$ International conference on electronics, machines and drives (PEMD 2020), March 2020, postponed to 15-17 Dec 2020, Nottingham, UK. http://dspace.lib.cranfield.ac.uk/handle/1826/16574

[12] F. Naseri, E. Farjah, T. Ghanbari, Z. Kazemi, E. Schaltz and J. Schanen, "Online Parameter Estimation for Supercapacitor State-of-Energy and Stateof-Health Determination in Vehicular Applications," in IEEE Transactions on Industrial Electronics, vol. 67, no. 9, pp. 7963-7972, Sept. 2020, doi: 10.1109/TIE.2019.2941151.

[13] M. S. Arulampalam, S. Maskell, N. Gordon and T. Clapp, "A tutorial on particle filters for online nonlinear/non-Gaussian Bayesian tracking," in IEEE Transactions on Signal Processing, vol. 50, no. 2, pp. 174-188, Feb. 2002, doi: 10.1109/78.978374.

[14] A. Fotouhi, D. J. Auger DJ, K. Propp, S. Longo, R. Purkayastha, L. O'Neill and S. Walus, "Lithium-Sulfur cell equivalent circuit network model parameterization and sensitivity analysis," IEEE Transactions on Vehicular Technology, vol. 66, no.9,pp. 7711-7721, 2017.

[15] M. Koot and J. T. B. A. Kessels and B. de Jager and W. P. M. H. Heemels and P. P. J. van den Bosch and M. Steinbuch, Energy management strategies for vehicular electric power systems, IEEE Transactions on Vehicular Technology, march 2005, 54(3),pp 771-782

[16] K. Li and K. J. Tseng, "An equivalent circuit model for state of energy estimation of lithium-ion battery," 2016 IEEE Applied Power Electronics Conference and Exposition (APEC), Long Beach, CA, 2016, pp. 3422-3430, doi: 10.1109/APEC.2016.7468359. 